\title{
Teste de Habilidades Sociais para crianças: evidências psicométricas de uma versão inicial
}

\author{
Daniel Bartholomeu1 - Centro Universitário Salesiano, Americana, Brasil \\ Marjorie Cristina Rocha da Silva - Faculdade Albert Einstein, Limeira, Brasil \\ José Maria Montiel - Faculdade Anhanguera de Jundiaí, Jundiaí, Brasil
}

\begin{abstract}
Resumo
Este artigo descreve o desenvolvimento de uma medida de avaliação das habilidades sociais em crianças do ensino fundamental. Foram investigadas 257 crianças que cursavam de segundo ao quarto ano, de escolas públicas de cidades do interior do estado de São Paulo, com idades entre 8 e 11 anos e média de 9 anos. A escala foi composta de 99 itens descritores de situações de relacionamento interpessoal em situações escolares. A análise de componentes principais com rotação varimax sugeriu uma estrutura de três fatores que explicaram $38,42 \%$ de variância. Os fatores foram civilidade e altruísmo (Alfa $=0,85)$, desenvoltura e auto-controle na situação de interação $($ Alfa=0,60) e assertividade de enfrentamento (Alfa $=0,47$ ). A aplicação do instrumento ocorreu de forma coletiva, realizada nas salas de aulas dos alunos e somente a aqueles cujos pais haviam autorizado préviamente. Esse instrumento avalia os fatores de habilidades sociais separando a dificuldade de expressão de cada uma das condutas, o que pode ser útil no planejamento de intervenções. Além disso, esses resultados denotam confiabilidade no uso do instrumento em futuras pesquisas.

Palavras-Chave: Habilidades sociais, Avaliação psicológica, Análise de Rasch, Análise fatorial, Funcionamento diferencial dos itens.
\end{abstract}

\section{Children's Social Skills Test: Psychometric evidences of the initial version}

\begin{abstract}
This article describes the development of a social skills measure for elementary school children. 257 children, attending in second to fourth grades in public schools in the interior of São Paulo State, aged from 8 to 11 years old (mean age 9 years, $\mathrm{DP}=0,77)$ were studied. The scale was composed by 99 items that described scholar interpersonal relationship situations. The data were analyzed by means of principal components analysis with varimax rotation and suggested a three factor structure explaining $38,42 \%$ of variance. The dimensions were altruism and civility (Alpha $=0,85$ ); self-control and resourcefulness in the social interaction (Alpha $=0,60)$ and coping assertivity (Alpha=0,47). The application of the instrument was made collectively, in the students' classrooms, and only to those students whose parents provided previous authorization. The instrument assesses social skill factors splitting the difficulty of behavior expression of each behavior, which can be useful in the planning of interventions. These data enables the instrument to be used in future research.

Keywords: Social skills, Psychological assessment, Rasch analysis, Factorial analysis, Differential item functioning.
\end{abstract}

Dizer que alguém é competente implica, basicamente, numa avaliação da qualidade da atuação dessa pessoa em uma dada tarefa. Nesses termos, o conceito de habilidade seria utilizado para designar capacidades específicas que conduziriam alguém na execução competente de alguma atividade ou tarefa. No caso específico das habilidades sociais, o termo "social" delimita o foco de interesse das habilidades, sendo um adjetivo dessas (Caballo, 2003).

Há duas suposições subjacentes aos conceitos de habilidades sociais. A primeira refere-se à habilidade social como um comportamento habilidoso, que é uma característica da personalidade do indivíduo, já a segunda como característica do desempenho numa situação de experiência pessoal (Mc Fall, 1982). Entende-se, de acordo com o primeiro conceito, que a habilidade social é uma qualidade inata do indivíduo, e o segundo, como um comportamento aprendido,

${ }^{1}$ Endereço para correspondência:

Rua Chá de Frade, 131, Mooca. São Paulo, SP

CEP - 03178-150.

E-mail: d_bartholomeu@yahoo.com.br resultante de experiências com diversas situações sociais.

A preocupação no estabelecimento de um modelo que possa explicar as habilidades sociais e, dessa forma, favorecer a condução de pesquisas e intervenções nesse aspecto tem suscitado interesse de pesquisadores (Argyle \& Kendon, 1967). Pesquisas como a de Trower (1982) procuraram estabelecer modelos gerais de habilidades sociais que, embora criticados, ampliaram o conhecimento acerca desse campo de trabalho, estabelecendo validade social para a pesquisa, na medida em que as conseqüências das condutas passaram a ser consideradas como critério para que a conduta fosse entendida como hábil socialmente. Componentes encobertos (como pensamentos, por exemplo) de comportamentos passaram a ser analisados no contexto específico em que ocorrem, ressaltando também a influência recíproca das pessoas nas situações de interação.

Tal modelo foi denominado, mais tarde, como interativo por Schroeder e Rakos (1983) uma vez que enfatizam a interação entre as variáveis ambientais e características pessoais na produção do 
comportamento social. Nesse contexto, uma resposta socialmente competente resulta de uma cadeia complexa de comportamentos que se iniciam na recepção de estímulos interpessoais relevantes, no processamento e levantamento das alternativas de resposta, das quais será selecionada a mais apropriada ao contexto, sendo finalizado com sua emissão (Robinson \& Calhoun, 1984; Caballo, 1998).

À parte desse modelo, não existe uma teoria geral que seja consensual entre os pesquisadores das habilidades sociais, sendo essa, uma dificuldade apontada no que concerne ao estudo dessa variável. Tal dificuldade é decorrente, para Caballo (2003), de uma controvérsia quanto à definição do termo, assim como da dependência do contexto cultural. Atrelado a isso, se aponta para a multiplicidade de dimensões e componentes que servem como indicadores dessa habilidade, tais como expressar opinião e emoções, habilidade de negar pedidos sem ofender os demais, defender seus direitos, habilidade de iniciar e manter interação, dentre outros.

Inúmeras tentativas de definição foram levadas a cabo ao longo dos anos buscando subsidiar a pesquisa nesse campo. Caballo (2003) define o comportamento socialmente hábil como um conjunto de condutas emitidas, em um determinado contexto interpessoal, que expressam sentimentos, desejos, opiniões e direitos, de forma adequada à situação, respeitando os comportamentos alheios e resolvendo os problemas imediatos do contexto, ao mesmo tempo em que minimiza a probabilidade de problemas futuros. Tal definição foi adotada nesse estudo. Apesar da ausência de consenso quanto à sua definição, conforme já assinalado, a literatura tende a concordar com o fato das habilidades sociais envolverem condutas interpessoais que incluem capacidades específicas, aprendidas e submetidas aos fatores ambientais e pessoais.

Não se pode afirmar com segurança em que época da vida ocorre o aprendizado das habilidades sociais, embora a infância seja um período importante nesse sentido. Inúmeros teóricos procuraram estabelecer um modelo explicativo do desenvolvimento das habilidades sociais. A esse respeito, Morrison (1990) esclarece que, as tendências temperamentais herdadas pelas crianças (variando da inibição à espontaneidade), mediaríam as formas de resposta em seu contexto social. Nessa perspectiva, as predisposições biológicas interagiríam com as experiências de aprendizagem e determinariam padrões consistentes do funcionamento social na infância.

Buck (1991) considera que o temperamento (expressividade emocional espontânea) em interação com o ambiente socioemocional interpessoal facilitaria a aprendizagem, sendo que a criança mais expressiva emocionalmente criaria um ambiente social e emocional mais agradável. Nesse sentido, o temperamento seria responsável pela expressividade da pessoa e esta traria implicações diversas para a vida social da mesma.

Bastante relevante também foi o estudo do temperamento infantil (ou inibição comportamental diante do não-familiar) de Kagan e Snidman (1991). Uma das contribuições desse estudo é que se acredita que crianças mais inibidas apresentarão menos oportunidades de aprender e praticar condutas sociais, recebendo menos reforços. Embora a predisposição biológica exerça um papel fundamental na determinação do comportamento social, fundamentalmente nos primeiros anos de vida, não se pode desconsiderar a maturidade e as experiências de aprendizagem (Kagan \& Snidman, 1991; Argyle, 1969).

Argumenta-se que dispor de um repertório de habilidades sociais mais elaborado contribuiria para o estabelecimento de relações mais harmoniosas com colegas e adultos na infância. Del Prette e Del Prette (2005) destacam ainda que não existem, no Brasil, dados disponíveis sobre a incidência de problemas interpessoais em crianças, mesmo estando presentes em queixas de pais, professores e em clínicas psicológicas.

A cultura apresenta normas e valores que influenciam as formas de relacionamento na medida em que define condutas aceitáveis, valorizadas e reprovadas em cada situação. Assim, a criança deve aprender habilidades sociais que atendam a diferentes demandas situacionais, articulando fatores pessoais, da situação e culturais, envolvendo sentimentos, pensamentos e ações (Gresham \& Elliot, 1990). A funcionalidade dessas condutas variaria em razão dos efeitos do seu desempenho. Del Prette e Del Prette (2001, 2005) destacam três tipos de resultados esperados no caso de um comportamento socialmente hábil, quais sejam, alcançar os objetivos imediatos; manter ou melhorar a qualidade dos relacionamentos, assim como a auto-estima.

As primeiras tentativas de se determinar habilidades sociais relevantes às crianças datam do final da década de 70 em que se afirmava a existência de 136 habilidades agrupadas em quatro conjuntos. Dez anos mais tarde, McGinnis, Goldstein, Sprafkin e Gershaw (1984) selecionaram 60 condutas e organizaram em cinco classes: sobrevivência em sala de aula; fazer amizades; lidar com sentimentos; alternativas à agressão; e lidar com o estresse (Stephens, 1992).

Tais categorias foram derivadas de observações feitas, principalmente no contexto escolar. Todavia, com o aperfeiçoamente dos instrumentos de avaliação, 
novas hipóteses empiricamente derivadas foram fornecidas e vieram corroborar a estrutura multidimensional dos conceitos, sugerindo novas dimensões. Um exemplo desse novo formato foi proposto por Gresham e Elliott (1990) em cinco classes, quais sejam, cooperação; asserção, que abrange pedir favores, convidar para atividades, dentre outros; responsabilidade; empatia; e autocontrole.

No Brasil, apesar de serem poucas as pesquisas que investigaram as habilidades sociais em crianças (Feitosa, 2003; Garcia, 2001; Martini, 2003, dentre outros), quatro classes dessas habilidades foram identificadas por Del Prette e Del Prette (2002). A primeira delas é empatia e civilidade; a segunda categoria assertividade de enfrentamento; autocontrole diante de frustrações ou reações negativas; finalmente, a participação que inclui habilidades de comprometerse com o contexto social mesmo quando as demandas não lhes são especificamente dirigidas.

Ponderando o fato de que o conhecimento do repertório social da criança é bastante escasso, Del Prette e Del Prette (2005) sugeriram sete classes de habilidades consideradas prioritárias no desenvolvimento de treinamentos e avaliações de habilidades sociais, acrescentando-se ao modelo anterior as categorias fazer amizades; solucionar problemas interpessoais; e habilidades sociais acadêmicas que abarcam seguir regras, prestar atenção, ignorar interrupções feitas pelos colegas, aguardar a vez para falar, oferecer, solicitar e agradecer ajuda, reconhecer a qualidade do outro e cooperar nas discussões.

No que concerne às medidas de avaliação, sabe-se produzir uma controvérsia entre estudiosos do tema, uma vez que não há estudos suficientes que atestem a qualidade da maior parte das medidas desenvolvidas. Tal constatação já era indicada por Bellack e Morrison (1982) com base na natureza questionável dos procedimentos de mensuração e do comportamento social, ou mesmo na dificuldade de um critério externo de validação e falta de consenso quanto à definição desse construto.

Destarte os procedimentos de entrevistas, medidas de auto-relato e outras formas de avaliação das habilidades sociais em crianças, alguns instrumentos padronizados foram identificados na literatura e merecem serem comentados. Exemplo disso é o estudo de LaFreniere e Dumas (1996) que estudaram a estrutura fatorial da versão reduzida da SCBE em 3 amostras americanas e uma de Quebec. A avaliação e produção de uma taxonomia das situações e tarefas que tem maior probabilidade de conduzir crianças em idade escolar a experienciar dificuldades sociais foi feita por Dodge, McClaskey e Feldman (1985). A estrutura fatorial demonstrou seis dimensões, a saber, entrada no grupo, resposta a provocações de pares, resposta a falhas, resposta ao sucesso, expectativa social e expectativa do professor. A seu turno, Stumme, Gresham e Scott (1983) desenvolveram um inventário de avaliação do comportamento social em crianças e os seis fatores identificados foram responsabilidade acadêmica, responsabilidade social, cooperação, comportamento adaptativo, participação e obediência. Numa outra perspectiva, Clark, Gresham e Elliott (1985) desenvolveram uma escala de 52 itens para medir as habilidades sociais de crianças pelo professor. A análise fatorial indicou quatro dimensões, performance acadêmica, iniciação social, cooperação e reforço dos pares.

No Brasil, o único procedimento para se mensurar as habilidades sociais em crianças em idade escolar compreende um sistema multimídia de avaliação proposto por Del Prette e Del Prette (2005). A aplicação desse instrumento é feita por meio da proposição de situações de interação social, ilustradas e apresentadas em formato multimídia ou em fichas, em que a criança deve responder em três formatos, quais sejam, a freqüência de ocorrência a dificuldade das situações e a adequação.

A correlação teste e reteste forneceu índices entre 0,57 e 0,50, indicando uma boa quantidade de variância de erro nas medidas efetuadas, sugerindo certa susceptibilidade às variações temporais. Também o alfa de Cronbach forneceu alguns índices satisfatórios (entre 0,31 e 0,67). A estrutura fatorial por dificuldade e freqüência demonstrou a existência de três e quatro fatores respectivamente (variância explicada para dificuldade, 34,52; variância explicada para freqüência, 29,72). As dimensões de análise são Empatia e Civilidade; assertividade de enfrentamento; autocontrole; ascendência sobre colegas, sugere habilidades de convencer colegas na resolução de necessidades pessoais; e participação, que envolve indicadores de comprometimento com o contexto social (Del Prette \& Del Prette, 2005).

Apesar de ter apresentado algumas propriedades psicométricas que o habilitem ao uso, foram detectadas algumas dificuldades quanto à sua aplicação nos estudos realizados por Del Prette e Del Prette (2005), já que, nem todas as escolas dispunham de uma infra-estrutura adequada e que comportasse a aplicação multimídia. Já a aplicação com fichas, embora mais fácil, pode apresentar problemas quanto à compreensão da tarefa, além de não terem sido feitos estudos específicos com este tipo de material.

O presente estudo justifica-se já que, dentre outras questões levantadas, o instrumento anteriormente citado é indicado para aplicação em 
adolescentes e crianças em idade escolar, e por considerar a realidade brasileira de que nem todas as escolas dispõem de computadores para a administração desse sistema; assim como o fato de que as qualidades psicométricas do mesmo ainda apresentam alguns problemas. Desse modo, considerou-se pertinente desenvolver um teste de habilidades sociais para crianças e pré-adolescentes, que pudesse ser aplicado em papel e com respostas objetivas, além de especificamente voltado para a avaliação das habilidades sociais no contexto escolar.

\section{Método}

\section{Participantes}

Foram investigadas 257 crianças que cursavam do segundo ao quarto anos de escolas públicas do interior do estado de São Paulo. A idade dos participantes variou de 8 a 11 anos, com média de 9 anos (DP=0,77), sendo 58,8\% meninas. Optaram-se por escolas públicas tanto de bairros mais pobres (cinco escolas) como outras de bairros com população mais bem abastada (três escolas) considerando que essas últimas, por terem maior acesso a uma diversidade maior de contextos e que poderiam apresentar habilidades sociais mais elevadas. Essa distribuição foi feita para se tentar obter uma distribuição bastante heterogênea em termos de habilidades sociais com vistas a testar se os itens do instrumento cobririam todos os seus níveis. Não houve seleção dos participantes em cada uma das escolas, já que se dependeu da assinatura dos termos de consentimento pelos pais dos alunos. Houve escolas que apresentaram desde seis crianças $(2,3 \%)$ até outras com 91 estudantes $(35,4 \%)$. A freqüência de crianças por escola e sua porcentagem é apresentada na Tabela 1.

Tabela 1 - Frequência e porcentagem de crianças por escola $(\mathrm{N}=257)$

\begin{tabular}{lcc}
\hline Escolas & Número de alunos & $\%$ \\
\hline 1 & 91 & 35,4 \\
2 & 14 & 5,4 \\
3 & 22 & 8,6 \\
4 & 13 & 5,1 \\
5 & 32 & 12,5 \\
6 & 35 & 13,6 \\
7 & 6 & 2,3 \\
8 & 44 & 17,1 \\
\hline Total & 257 & 100,0 \\
\hline
\end{tabular}

\section{Instrumentos}

Teste de Habilidades Sociais

Adotou-se como pressuposto na construção dos itens que o comportamento socialmente hábil deve ser considerado sempre em razão de um contexto específico e avaliado por suas conseqüências, conforme a definição de Caballo (2003). Outro aspecto levado em conta foi que as características apontadas seriam específicas à pessoa e a uma dada situação. Assim, alguns indicadores foram propostos pensando-se no contexto escolar e nas condutas que são socialmente eficazes.

Os comportamentos considerados foram $\mathrm{O}$ fazer elogios, aceitar elogios, fazer pedidos, expressar amor, agrado e afeto, iniciar e manter conversações, defender os próprios direitos, recusar pedidos, expressar opiniões pessoais, inclusive o desacordo, expressar incômodo, desagrado ou enfado justificados, pedir a mudança de conduta do outro, desculpar-se ou admitir ignorância, enfrentar as críticas, dar reforço ao outro, dar e receber retroalimentação, regular a entrada e saída de grupos sociais e falar em público. Todos esses comportamentos foram evidenciados em estudos sobre as habilidades sociais (Furnham \& Henderson, 1984; Rathus, 1975; Caballo, 2003; Del Prette \& Del Prette, 2005), e por esta razão foram considerados na elaboração desse instrumento.

As questões propostas descrevem comportamentos realizados com o intuito de otimizar os ganhos e minimizar perdas nas relações sociais. Além disso, essas condutas visam à manutenção da própria integridade, bem como a produção de uma sensação de domínio, o que caracteriza uma eficácia no respeito a si próprio. Uma análise preliminar dos 120 itens reduziu a 99 o número de questões do instrumento, sendo excluídas aquelas muito parecidas ou que foram rejeitadas pelos avaliadores por apresentarem problemas em sua estrutura, dando duplo sentido em interpretações, ou mesmo por não serem pertinentes à avaliação das habilidades sociais.

As categorias de resposta foram apresentadas em uma escala likert de quatro pontos (nunca, com 
pouca freqüência, freqüentemente, e sempre) $\mathrm{e}$ atribuídos valores de 1 a 4 respectivamente, sendo que alguns itens foram pontuados de forma inversa, já que a conduta socialmente hábil estava definida no oposto do que a frase propunha. Os itens que tiveram suas pontuações invertidas foram 22 e seguem três exemplos de itens dessa natureza.

"Não consigo fazer perguntas a meus professores."

"Não gosto de ser criticado por meus amigos de classe mesmo quando estou errado"

"Quando chego num grupo que ninguém me conbece fico com vergonha."

\section{Procedimento}

A aplicação do instrumento ocorreu de forma coletiva, realizada nas salas de aulas dos alunos e somente a aqueles cujos pais haviam autorizado préviamente pela assinatura de um termo de consentimento livre e esclarecido (número de projeto comitê de ética 424-2010) Foi, inicialmente explicado às crianças de que se tratava a pesquisa e como responder ao instrumento. As questões foram lidas uma a uma pelo pesquisador que aguardava a resposta antes de prosseguir a coleta. As crianças que não desejavam prosseguir tinham essa liberdade, sendo liberadas da aplicação.

\section{Resultados}

Tabachinick e Fidell (1996) destacam que para a realização adequada $\mathrm{da}$ análise fatorial seriam necessários entre cinco e 10 pessoas por item. Todavia, Guadagnoli e Velicer (1988) indicaram em seu estudo por simulação de Monte Carlo, que o tamanho da amostra não é um fator importante na determinação da estabilidade dos fatores encontrados em uma análise fatorial. Também os estudos de Velicer e Fava (1998) e MacCallum, Widaman, Zhang e Hong (1999) sugerem que a adequação da solução fatorial não pode ser predita pela proporção participantes-variáveis. Ao invés disso, a precisão dos fatores depende da a) raiz quadrada do tamanho da amostra, portanto da estabilidade da matriz de correlações, b) o número de variáveis que definem um fator com cargas fatoriais elevadas (três no mínimo) e c) a magnitude das cargas fatoriais em um fator, relacionada à comunalidade das variáveis incluídas na análise. Ainda assim, é sugerido que os pontos fortes em uma área podem compensar as fraquezas em outras.

$\mathrm{Na}$ análise fatorial dos itens da escala, a medida de adequação da amostra de Kaiser-Meyer-Olkin $(\mathrm{KMO}=0,80)$ e o teste de esfericidade de Bartlett $\left(X^{2}\right.$ $=1010,69, \mathrm{gl}=253, p=0,000)$ sugeriram a possibilidade de extração de mais de um fator nos itens da escala de habilidades sociais. A análise anti-image, que indica valores negativos da correlação parcial entre os itens, mostrou que todos os coeficientes foram nulos, sugerindo a existência de mais de um fator (Tabachinick \& Fidell, 1996). O gráfico de sedimentação encontra-se na Figura 1.

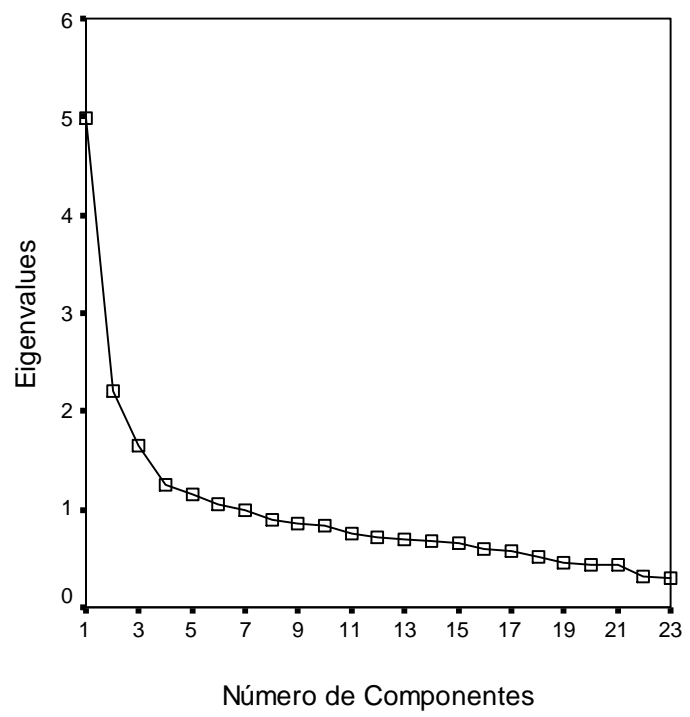

Figura 1. Gráfico de Sedimentação do THAS-C $(N=257)$

Nessa figura, o eixo $\mathrm{Y}$ apresenta os valores dos eigenvalues enquanto no eixo $\mathrm{X}$ está o número de fatores. Sugerido por Cattell (1966), o uso desse gráfico é um dos procedimentos para a identificação da 
quantidade de fatores a serem extraídos de um conjunto de itens. Na Figura 1, observa-se uma maior descontinuidade na curva do gráfico quando o terceiro fator é representado. Dessa forma, seguiu-se a indicação desse procedimento, estudando-se uma estrutura de três fatores.

Considerando-se que a construção dos itens pautou-se em diferentes situações acadêmicas em que o aluno poderia manifestar sua competência social, assim como diferentes categorias de análise do comportamento socialmente eficaz, julgou-se mais pertinente a utilização da rotação Varimax, já que se esperava a extração de fatores independentes entre si. Da mesma forma, esse método de rotação é o que produz um padrão mais invariante (Kaiser, 1970). O método de extração dos fatores aplicado foi o de Componentes Principais, já que se optou por se extrair uma quantidade mínima de fatores que explicasse a maior porcentagem possível da variância dos itens (Hair, Anderson, Tatham \& Black, 1995).

Apesar das inúmeras regras para identificação do número adequado de fatores a serem extraídos em análise fatorial, deve-se questionar sempre a compreensividade dos fatores extraídos, já que muitas das estruturas identificadas podem não fazer sentido do ponto de vista da teoria que embasou a construção dos itens. Desse modo, buscou-se uma solução fatorial que apresentasse características psicométricas adequadas e que fornecesse uma coerente interpretação teórica.

A estrutura de três fatores mencionada (fornecida pelo gráfico de sedimentação) demonstrou ser a que melhor representa os construtos teóricos idealizados na construção dos itens, assim como apresentaram os resultados mais adequados psicometricamente. Considerando que se desejava uma escala mais compacta e que também utilizasse os itens com cargas fatoriais mais altas nos fatores, ou, em outros termos, que fossem mais altamente correlacionados a eles, utilizou-se como regra de inclusão de itens nos fatores aqueles que tivessem saturação acima de 0,40 . Os resultados da análise de componentes principais com os níveis de dificuldade dos itens por Rasch e seus respectivos ajustes a esse modelo, além de coeficientes de consistência interna são apresentados na Tabela 2.

Tabela 2 - Estatísticas dos Fatores e itens por análise de componentes principais, rotação varimax com normalização de Kaiser, por Rasch e consistência interna do THAS-C

\begin{tabular}{|c|c|c|c|c|c|c|c|}
\hline \multirow[t]{2}{*}{ Itens } & \multicolumn{3}{|c|}{ Componentes } & \multirow{2}{*}{$h^{2}$} & \multirow{2}{*}{ Dificuldade } & \multirow{2}{*}{ Infit } & \multirow{2}{*}{ Outfit } \\
\hline & 1 & 2 & 3 & & & & \\
\hline Item 68 & 0,73 & & & 0,53 & $-0,72$ & 1,02 & 0,87 \\
\hline Item 72 & 0,68 & & & 0,48 & $-0,55$ & 0,90 & 0,85 \\
\hline Item 41 & 0,66 & & & 0,44 & $-0,43$ & 0,74 & 0,69 \\
\hline Item 67 & 0,64 & & & 0,44 & $-0,45$ & 0,78 & 0,73 \\
\hline Item 84 & 0,64 & & & 0,41 & $-0,39$ & 0,78 & 0,76 \\
\hline Item 76 & 0,61 & & & 0,38 & $-0,27$ & 0,81 & 0,77 \\
\hline Item 11 & 0,58 & & & 0,34 & $-0,79$ & 0,94 & 0,83 \\
\hline Item 51 & 0,56 & & & 0,33 & $-0,30$ & 0,82 & 0,81 \\
\hline Item 55 & 0,56 & & & 0,35 & $-0,45$ & 0,96 & 0,94 \\
\hline Item 83 & 0,55 & & & 0,32 & $-0,40$ & 0,74 & 0,72 \\
\hline Item 30 & 0,52 & & 0,45 & 0,49 & $-0,50$ & 0,84 & 0,79 \\
\hline Item 77 & 0,51 & & & 0,43 & $-0,29$ & 0,75 & 0,75 \\
\hline Item 36 & 0,48 & & & 0,25 & $-0,69$ & 0,99 & 0,89 \\
\hline Item 75 & & 0,68 & & 0,47 & 0,60 & 1,14 & 1,18 \\
\hline Item 27 & & 0,67 & & 0,46 & 0,81 & 1,38 & 1,47 \\
\hline Item 81 & & 0,57 & & 0,37 & 0,39 & 1,06 & 1,07 \\
\hline Item 70 & & 0,52 & & 0,29 & 0,47 & 1,14 & 1,18 \\
\hline Item 97 & & 0,51 & & 0,28 & 0,43 & 1,14 & 1,16 \\
\hline Item 66 & & 0,45 & & 0,25 & 0,13 & 1,12 & 1,14 \\
\hline Item 7 & & & 0,70 & 0,50 & 0,53 & 0,98 & 0,97 \\
\hline Item 85 & & & 0,60 & 0,41 & 0,47 & 1,05 & 1,05 \\
\hline Item 15 & & & 0,59 & 0,35 & 0,14 & 0,99 & 0,98 \\
\hline Item 54 & & & 0,51 & 0,30 & 0,14 & 0,88 & 0,88 \\
\hline Alfa de Cronbach & 0,85 & 0,60 & 0,47 & & & & \\
\hline Duas Metades Spearman-Brown & 0,84 & 0,60 & 0,43 & & & & \\
\hline Total de Variância Explicada & 20,76 & 9,33 & 8,33 & & & & \\
\hline Eigenvalue & 4,78 & 2,15 & 1,92 & & & & \\
\hline
\end{tabular}


Nessas condições restaram 23 itens, distribuídos em três fatores, explicando $38,42 \%$ da variância. $O$ primeiro fator apresentou 13 itens e foi denominado Civilidade e altruísmo, explicando 20,76\% de variância. $O$ segundo fator, Desenvoltura e autocontrole na interação social, ficou com 6 itens e explicou 9,33\% de variância e seus itens devem ser pontuados de forma inversa. Finalmente, a terceira dimensão, com 4 itens recebeu a denominação de Assertividade com enfrentamento e explicou $8,33 \%$ de variância.
Quanto ao nível de dificuldade, observou-se que os indicadores avaliam habilidades sociais medianas, embora o nível de dificuldade dos itens aumente de um fator para o outro, sendo que os indicadores do fator 1 apresentam maiores concordâncias, enquanto os do fator 3 menor. Também o ajuste dos itens restantes da escala por Rasch evidenciou que nenhum desses ultrapassou o limite de 1,5 considerado aceitável (Linacre, 2002). Somente o item 27 apresentou valores de infit e outfit acima de 1,3, mas, considerando seu valor na estrutura fatorial, optou-se por não retirá-lo.

Tabela 3 - Parâmetros de ajuste de itens e das pessoas $(N=257)$

\begin{tabular}{lccrrrrcc}
\hline \multirow{2}{*}{$\underline{\text { Parâmetros }}$} & \multicolumn{4}{c}{ Itens } & \multicolumn{4}{c}{ Pessoas } \\
\cline { 2 - 9 } Média & Dificuldade & Infit & Outfit & Erro & Habilidade & Infit & Outfit & Erro \\
DP & 0,00 & 1,00 & 1,00 & 0,06 & 0,35 & 1,02 & 1,01 & 0,10 \\
Máximo & 0,34 & 0,17 & 0,19 & 0,01 & 0,28 & 0,37 & 0,37 & 0,01 \\
Mìnimo & 0,89 & 1,38 & 1,47 & 0,09 & 1,33 & 2,12 & 2,18 & 0,20 \\
\hline
\end{tabular}

A soma dos indicadores nos fatores 1, 2 (após a inversão) e 3 forneceu o índice de habilidade social geral. A partir desses dados, foram calculados os coeficientes de consistência interna dos fatores por meio das medidas Alfa de Cronbach e duas metades de Spearman-Brown. O resultado da última subescala forneceu índices baixos em decorrência do número reduzido de itens. Para se corrigir o efeito da quantidade de itens pode-se utilizar a fórmula de profecia Spearman-Brown. Essa análise supõe que se houvessem itens com conteúdos semelhantes aos de uma subescala qualquer cuja quantidade é pequena, mas estivessem em uma quantidade correspondente à escala toda, o coeficiente seria aumentado. Esse método forneceu índices de 0,84 para Alfa e 0,82 para duas metades. Desse modo, os dados de precisão por esses dois métodos indicaram índices satisfatórios. Assim, os fatores do THAS-C apresentaram características psicométricas adequadas para a avaliação de seu constructo.

Optou-se por se executar a prova de correlação produto-momento de Pearson entre os escores produzidos pelos fatores para se verificar se estaríam associados. Os índices (embora alguns deles significativos) foram nulos, mantendo-se dentro do esperado uma vez que, optou-se por uma rotação ortogonal. (Altruísmo e desenvoltura; $r=0,80, p=0,257$; Altruísmo e Assertividade; $\quad r=0,19, \quad p=0,007$ Desenvoltura e Assertividade; $r=0,16, p=0,023)$. A média de dificuldade dos itens para o Fator 1 foi $-0,48$ para o fator 2, 0,54 e no terceiro fator, 0,32.

Alguns dos itens com funcionamento diferencial apresentaram saturações altas nos fatores indicados. Os resultados da análise de funcionamento diferencial de itens por sexo revelaram que somente cinco desses demonstraram favorecer um ou outro desses grupos.

Tabela 4 - Medidas de DIF por Sexo por Item com Diferença Significativa ( $N=257)$

\begin{tabular}{lcrrrrr}
\hline Item & Sexo & $\begin{array}{c}\text { DIF } \\
\text { Acrescido }\end{array}$ & Sexo & $\begin{array}{c}\text { DIF } \\
\text { acrescido }\end{array}$ & Mudanca & $t$ \\
\hline Itens mais freqüentes ao sexo masculino & & & & & & \\
\hline Hs27 & 1 & 0,49 & 2 & 1,07 & $-0,58$ & $-4,38$ \\
Hs75 & 1 & 0,38 & 2 & 0,75 & $-0,37$ & $-2,91$ \\
Hs87 & 1 & 0,25 & 2 & 0,62 & 0,37 & 2,95 \\
\hline Itens mais freqüentes ao sexo feminino & & & & & & \\
\hline Hs29 & 1 & 0,23 & 2 & $-0,30$ & 0,53 & 4,11 \\
\hline Hs68 & 1 & $-0,45$ & 2 & $-0,96$ & 0,51 & 3,00 \\
\hline
\end{tabular}


Embora tenham apresentado viés, o fato de terem obtido altas saturações nesses fatores é um dado que deve ser considerado, já que a relevância desses indicadores para o constructo mensurado em cada fator, assim como sua contribuição em cada um pode ser indicada como validade para esses itens (Anastasi \& Urbina, 2000).

\section{Discussão}

A insuficiência de pesquisas que atestassem as qualidades psicométricas de medidas de avaliação das Habilidades Sociais em crianças foi uma das lacunas que incitaram o desenvolvimento do presente estudo. Atrelado a isso, no Brasil, o único instrumento dessa natureza compreende um sistema multimídia, desenvolvido por Del Prette e Del Prette (2001), indicado para aplicação no final da adolescência e adultos. Desse modo, considerou-se pertinente desenvolver um teste de habilidades sociais voltado para crianças e pré-adolescentes, que pudesse ser aplicado no formato lápis e papel, com respostas objetivas, e especificamente voltado ao contexto escolar.

Há que se considerar também que não foram identificados trabalhos que empregassem a Teoria de Resposta ao Item para se estudar indicadores de habilidades sociais. Nessa pesquisa, evidenciaram-se poucos itens desajustados (cerca de 7\%) e cerca de $40 \%$ das pessoas com problemas nas medidas de ajuste, o que sugere que houve sujeitos com altos níveis de habilidades e que não concordaram com alguns itens mais fáceis (que apresentaram altas concordâncias pela maioria das crianças) e vice-versa, devendo ser mais investigada a compreensão das crianças no que diz respeito à tarefa solicitada.

Além disso, seria interessante uma re-aplicação do teste numa amostra de crianças com habilidades sociais mais baixas, a fim de prover mais informações sobre itens que avaliam essas habilidades, embora a maior parte dos itens tenha apresentado dificuldade entre +1 e -1 logit, intervalo que avalia as pessoas com mais precisão.

A análise fatorial realizada sugeriu a existência de três fatores que explicaram aproximadamente 38\% de variância (23 itens) e que foram denominados Civilidade e Altruísmo, Desenvoltura e autocontrole na interação social e Assertividade com enfrentamento. Analisando mais detidamente os itens em cada um dos fatores, ficou evidente que a análise fatorial separou os itens, basicamente, por nível de dificuldade. Ou, ainda, sugere-se a reaplicação dos itens em sua versão final para verificar se a estrutura fatorial é mantida, a fim de respaldar os resultados ora obtidos. Tal reaplicação assume relevância ao se pensar em acrescentar itens para se aumentar a variância explicada, assim como os coeficientes Alfa. De fato, os itens do teste demonstraram boas qualidades mas poderíam ser ampliados em uma outra versão para implementar suas características psicométricas. Da mesma forma sua aplicação com outros instrumentos como o CBCL seria importante, tanto no processo de seleção de itens como para estabelecer notas de corte para avaliação da saúde mental de crianças, por exemplo. Também outros critérios como o teste sociométrico (que avalia a aceitação e rejeição por pares em um grupo) poderia ser empregado para se estabelecer que características de habilidades sociais crianças em idade escolar apresentam que aumenta a probabilidade de interação com os demais, de forma a ser uma ferramenta no planejamento de intervenções escolares, considerando a importância da interação social no processo de aprendizagem.

No que concerne à relação do número de sujeitos pelo de variáveis em questão, o número mínimo de variáveis em um fator determinado foi cumprido. Também, as comunalidades encontradas por alguns itens tiveram índices abaixo do patamar 0,50, estabelecido pelos manuais (Hair, Anderson, Tatham \& Black, 1995). Quando as comunalidades são consistemente abaixo desse valor (o caso desse estudo) para garantir uma boa recuperação dos fatores da população, a superestimação dos fatores e amostras grandes (acima de 100 sujeitos) são necessárias. Nesse estudo, a estrutura identificada de três fatores com pelo menos 4 indicadores em cada fator e o tamanho da amostra acima de 100, cumprem o critério de MacCallum e colaboradores (1999) sugerindo e fazendo possível uma boa estimação dos fatores populacionais.

De uma perspectiva mais teórica, os instrumentos de avaliação das habilidades sociais em crianças, disponíveis na literatura, encontraram fatores mais pontuais. No trabalho de LaFreniere e Dumas (1996), por exemplo identificou-se três fatores denominados de competência social, ansiedade e timidez e agressão e raiva, indicando, basicamente, sentimentos que as crianças podem ter nas situações sociais, dado esse não fornecido especificamente pelo THAS-C, embora alguns itens reflitam também tais aspectos. Porém, é necessário ressaltar que essas dimensões de LaFreniere e Dumas (1996) não consideram o contexto em que a criança está inserida, assim como as conseqüências dos comportamentos, características imprescindíveis para se considerar um comportamento como socialmente hábil, enquanto que o THAS-C contempla tais aspectos em suas avaliações. 
A primeira dimensão, Civilidade e altruísmo, abrange habilidades como agradecer elogios, pedir desculpas, ajudar os amigos, elogiá-los, expressar sentimentos positivos aos pares, ser educado ao manifestar uma opinião. No segundo fator, Desenvoltura e autocontrole na interação social, seus indicadores devem ser invertidos para indicarem tais aspectos. As situações propostas sugerem situações negativas em que a criança é exposta a situações novas, desconhecidas ou que podem provocar constrangimento como receber críticas, falar para a sala toda, encerrar uma conversa, apresentar-se a um grupo de desconhecidos ou perguntar-lhes algo. Finalmente, a terceira dimensão, recebeu a denominação de Assertividade com enfrentamento. As habilidades propostas incluem demonstrar desagrado, defender seus direitos e opiniões, resistir à pressão do grupo, afirmando sua auto-estima, sob o risco de uma reação indesejável por parte do interlocutor. Esses fatores foram similares aos sugeridos por Del Prette e Del Prette (2005)

O trabalho de Dodge, McClaskey e Feldman (1985) também identificou seis fatores, denominados entrada no grupo, resposta a provocações de pares, resposta a falhas, resposta a sucesso, expectativa social e expectativa do professor. Aspectos como respostas a provocações de colegas e a falhas ou sucesso estão contidos no fator Desenvoltura e autocontrole do THAS-C. Nesse e nos parágrafos de cima e abaixo são feitas considerações interpretativas que o presente instrumento agrega em relação aos demais, sugestão dada pelos pareceristas. Uma parte dessas considerações que julgou-se faltante realmente (acatando-se à sugestão do parecerista) também foi acrescentada acima.

No Brasil, o sistema multimída de Del Prette e Del Prette (2005) evidenciou cinco fatores, quais sejam, empatia e civilidade, assertividade de enfrentamento, ascendência sobre os colegas, autocontrole e participação. Algumas dimensões foram separadas de forma similar como é o caso de empatia e civilidade, assertividade de enfrentamento e autocontrole. Todavia, a estrutura de cinco dimensões proposta no estudo de Del Prette e Del Prette (2005) explicou $34,52 \%$ de variância, enquanto os três fatores do THAS-C explicaram 38,42\%. Além disso, a medida Alfa de Cronbach indicou índices mais satisfatórios variando de 0,47 a 0,85 , enquanto no sistema multimídia variou de 0,31 a 0,67 .

\section{Considerações Finais}

De modo geral, esse instrumento fornece uma informação diferenciada que talvez seja desejável de ser obtida por pesquisadores e psicólogos que trabalham com essa variável. Ao se hierarquizar a dificuldade dos itens, identificando quais condutas são mais fáceis de se apresentar no contexto de interação social, possibilitase planejar intervenções para pessoas que apresentam baixa habilidade social, apresentando inicialmente condutas mais fáceis da pessoa emitir, para então passar às comportamentos mais complexos, permitindo uma avaliação precisa do repertório comportamental da criança, possibilitando um escalonamento comportamental, o que convida a novas pesquisas. Essa informação é uma das mais relevantes nesse teste, conforme a sugestão do parecerista

Nesse contexto, pode-se sugerir que comportamentos relacionados à civilidade e altruísmo são mais fáceis de serem apresentadas pelas crianças, enquanto condutas referentes à desenvoltura e autocontrole são as mais difíceis de serem evidenciadas. Comportamentos assertivos com enfrentamento apresentaram um nível intermediário de dificuldade em relação aos outros dois fatores. De fato, a possibilidade de uma reação indesejável por parte dos interlocutores pode provocar uma maior dificuldade para a produção de comportamentos assertivos. Todavia, aparentemente, apresentar desenvoltura perante a situações novas e controlar os sentimentos ao ter de se expor ou apresentar-se à outras pessoas desconhecidas, produz uma maior dificuldade às crianças, em detrimento dos demais tipos de comportamentos avaliados pela escala.

Em outros termos, o dado fornecido por esse instrumento não apenas indica quais as condutas específicas de habilidade social estão sendo medidas mas fornece um índice da capacidade social do indivíduo, sendo que os itens carregados em cada um dos fatores também abrangem as categorias comportamentais mencionadas na literatura. Ao mesmo tempo, identifica os comportamentos interpessoais como fazer pedidos ou recusá-los e que não é identificado nos demais instrumentos (Caballo, 2003; Trower, 1982). Considerando ainda que se possui o nível de dificuldade de todos os itens avaliados, não só os que permaneceram na escala final, novos estudos poderíam investigar formas paralelas de investigação com essa escala pela análise dos itens restantes da análise fatorial (que não se congregaram em nenhum dos fatores encontrados)

Por fim, resta destacar que os índices de precisão foram satisfatórios. Integrando esse dado com os demais já mencionados, permite atestar a boa qualidade do instrumento constituído, habilitando-o para o uso em pesquisas. Novas investigações podem ser conduzidas no sentido de verificar se a estrutura fatorial desse instrumento é mantida sob outras 
condições, ou mesmo novos estudos de precisão como teste-reteste e validade discriminativa.

\section{Referências}

Achenbach, T.M. \& Edelbrock, C.S. (1978). The classification of child psychopathology: A review and analysis of empirical efforts. Psychological Bulletin, 85, 1275-1301.

Anastasi, A., \& Urbina, S. (2000). Testagem Psicológica (7a ed.). Porto Alegre: Artes Médicas.

Argyle, M. \& Kendon, A., (1967). The experimental analysis of social performance. Advances in Experimental Social Psychology, 3, 55-98.

Argyle, M. (1969). Social Interaction. London: Methuen.

Bellack, A. S. \& Morrison, R. L. (1982). Interpersonal dysfunction. En A. S. Bellack, M. Hersen y A. E. Kazdin (comps.), International bandbook of behavior modification and therapy. Nueva York, Plenum Press.

Buck, R. (1991). Temperamento, habilidades sociais, bem como a comunicação de emoção: A-interacionista perspectiva de desenvolvimento. Em D. Gilbert e JJ Conley (Eds. Personalidade), Habilidades sociais, $e$ Psicopatologia: Uma Abordagem de Diferenças Individuais. New York: Plenum, 85-106.

Caballo, V. E. (1998). Manual para el tratamiento cognitivoconductual de los transtornos psicológicos, vol. 2: Formulation clinica, medicina conductual y trastornos de relación. Madrid, Siglo XXI.

Caballo, V.E. (2003). Manual de Avaliação e Treinamento das Habilidades Sociais. Santos Editora. São Paulo.

Cattell, R. B. (1966). The meaning and strategic use of factor analysis. Em R. B. Cattell (Org.), Handbook of multivariate experimental psychology (pp. 174 -243). Chicago: Rand McNally.

Clark, L.; Gresham, F.M.; \& Elliott, S.N. (1985). Development and validation of a social skills assessment measure: The TROSS--C. Journal of Psychoeducational Assessment, 3(4) 347-356.

Del Prette, Z.A.P. \& Del Prette, A. (2001). Inventário de habilidades sociais: manual de aplicação, apuração $e$ interpretação. São Paulo. Casa do Psicólogo.

Del Prette, Z.A.P. \& Del Prette, A. (2002). Avaliação de habilidades sociais de crianças com um inventário multimídia: indicadores sociométricos associados à freqüência versus dificuldade. Psicologia em estudo, 1, 61-73.

Del Prette, Z.A.P. \& Del Prette, A. (2005). Sistema Multimídia de Habilidades Sociais de Crianças: (SMHSC-Del Prette) manual. São Paulo. Casa do Psicólogo.

Dodge, K.A.; McClaskey, C.L.; \& Feldman, E. (1985). Situational approach to the assessment of social competence in children. Journal of Consulting and Clinical Psychology, 53(3), 344-353.

Feitosa, F.B. (2003). Relação família-escola: Como pais e professores avaliam e reagem ao repertório social de crianças com e sem dificuldades de aprendizagem. Dissertação de Mestrado. Programa de Pós-Graduação em Educação Especial. Universidade Federal de São Carlos.

Furnham, A. \& Henderson, M. (1984). Assessing assertiveness: A content and correlational analysis of five assertiveness inventories. Behavioral Assessment, 6, 79-88.

Garcia, F.A. (2001). Ivestigando diferentes indicadores de empatia em meninos e sua relação com a empatia e ações educativas dos pais. Dissertação de Mestrado. Programa de Pós-Graduação em Psicologia. FFCLRP - Universidade de São Paulo.

Gresham, F.M. \& Elliott, S.N. (1990). Social Skills Rating System. Circle Pines, MN: American Guidance Service.

Guadagnoli, E. \& Velicer, W. F. (1988). Relation of sample size to the stability of component patterns. Psychological Bulletin, 103(2), 265-275.

Hair, J.F.; Anderson, R.E.; Tatham, R.L.; \& Black, W.C. (1995). Multivariate Data analysis with readings. Prentice-hall international, New Jersey.

Kagan, J., \& Snidman, N. (1991). Infant predictors of inbibited and uninbibited profiles. Prentice-hall international, New Jersey.

Kaiser, H.F. (1970). A second-generation Little Jiffy. Psychometrika, 35, 401-415.

Kazdin, A.E \& Weisz, J.R. (Orgs.) (2003). Evidence-based psychotherapies for children and adolescent. New York: The Guilford Press.

LaFreniere, P.J.; \& Dumas, J.E. (1996). Social competence and behavior evaluation in children ages 3 to 6 years: The short form (SCBE-30). Psychological Assessment, 8(4), 369-377.

Linacre J. M., (2002).What do Infit and Outfit, MeanSquared and Standardized mean? Rasch Measurement Transactions, 16(2), 878. Disponível em: $<$ http://209.238.26.90/rmt/rmt82a.htm>.

Acessado em: 10 jun. 2006.

Loeber, R. (1991). Antisocial behavior: More enduring than changeable? Journal of the American Academy of Child and Adolescent Pschiatry, 30, 393-397.

MacCallum, R. C., Widaman, K. F., Zhang, S., \& Hong S. (1999). Sample size in factor analysis. Psychological Methods, 4, 84-99.

MacCallum, R.C., Widaman, K.F., Zhang, S., \& Hong, S. (1999). Sample size in factor analysis. Psychological Methods, 4, 84-99.

McFall, R. (1982). A review and reformulation of the concept of social skills. Behavioral Assessment, 4, 1-33. 
Martini, M.L. (2003). Relaçoes professor-aluno e rendimento acadêmico: Uma análise das crencas, sentimentos $e$ desempenhos de professores e alunos do Ensino fundamental. Tese de doutorado. Programa de Pós-Graduação em Psicologia. FFCLRP - Universidade de São Paulo.

McGinnis, E.; Goldstein, A.P.; Sprafkin, R.P. \& Gershaw, N.J. (1984). Skillstreaming the elementary school child: A guide for teaching prosocial skills. Champaign: Illinois: Research Press.

Morrison, D. F. (1990), Multivariate Statistical Methods. McGraw-Hill, New York.

Rathus, S. A.(1975). Principles and practices of assertive training: An eclectic overview. The Counseling Psychologist, 5, 9-20.

Robinson, W. L. \& Calhoun, K. S. (1984). Assertiveness and cognitive processing in interpersonal situations. Journal of Behavioral Assessment, 6, 81-96.

Schroeder, H. E. \& Rakos, R. F. (1983). The identification and assessment of social skills. Em R. Ellis y D. Whittington (comps.). New directions in social skill training, Londres, Croom Helm.
Stephens, T.M. (1992). Social skills in the classroom. Odessa (FL): Psychology Assessment Resources.

Stumme, V.S.; Gresham, F.M.; \& Scott, N.A. (1983). Dimensions of children's classroom social behavior: A factor analytic investigation. Journal of Behavioral Assessment, 5(3), 161-177.

Tabachinick, B. G. \& Fidell, L. S. (1996). Using multivariate statistics. New York: HarperCollins. Psychological Science, 2, 40-44.

Trower, P. (1982). Towards a generative model of social skills: A critique and synthesis. Em J. P. Curran y P. M. Monti (comps.), Social skills training: A practical bandbook for assessment and treatment. Nueva York, Guilford Press.

Velicer, W. F., \& Fava, J. L. (1998). Effects of variable and subject sampling on factor pattern recovery. Psychological Methods, 3, 231-251.
Recebido em marco de 2010 Reformulado em agosto de 2010 Aprovado em outubro de 2010

Sobre os autores:

Daniel Bartholomeu é psicólogo formado pela Universidade São Francisco, tendo realizado seu mestrado nessa mesma instituição. Atualmente é doutorando em Avaliação Psicológica também pela Universidade São Francisco e atua como docente na Faculdade Anhanguera de Jundiaí e no Centro Universitário Salesiano de Americana. Membro do grupo de pesquisa do Laboratório de Avaliação Psicológica e Educacional da Universidade São Francisco - LabAPE, do LEPESPE, Laboratório de Estudos e Pesquisas em Psicologia do Esporte da UNESP-Rio Claro e coordenador do Laboratório de Psicodiagnóstico e Neurociências Cognitivas - LaPsiNeCo do Centro Universitário Salesiano de Americana.

Marjorie Cristina Rocha da Silva é Psicóloga, Mestre e Doutoranda em Psicologia pelo Programa de PósGraduação Stricto Sensu da Universidade São Francisco. Membro do grupo de pesquisa do Laboratório de Avaliação Psicológica e Educacional - LabAPE da Universidade São Francisco e do Laboratório de Psicodiagnóstico e Neurociências Cognitivas - LaPsiNeCo do Centro Universitário Salesiano de Americana. Atua como docente na Faculdade Albert Einstein de Limeira.

José Maria Montiel possui graduação em Psicologia, mestrado em Avaliação Psicológica em Contextos de Saúde Mental. Doutorando em Psicologia (Avaliação Psicológica em Contextos de Saúde Mental) pela Universidade São Francisco. Atualmente é bolsista Capes pela Universidade São Francisco. Tem experiência na área de Psicologia, com ênfase em Psicologia Cognitiva e Neuropsicologia, atuando principalmente nos seguintes temas: avaliação psicológica, avaliação neuropsicológica com ênfase em transtornos mentais e problemas de aprendizagem (leitura escrita), desenvolvimento de instrumentos para avaliação psicológica e neuropsicológica e intervenções psicológicas. Membro do Laboratório de Psicodiagnóstico e Neurociências Cognitivas. Atua como docente da Faculdade Anhanguera de Jundiaí. 